QCD Evolution Workshop 2014

International Journal of Modern Physics: Conference Series

Vol. 37 (2015) 1560065 (8 pages)

(C) The Author

DOI: $10.1142 / \mathrm{S} 2010194515600654$

\title{
Studies of Inclusive Hadron Production at BABAR
}

\author{
Ray F. Cowan* \\ Laboratory for Nuclear Science, Massachusetts Institute of Technology, \\ 77 Massachusetts Avenue, \\ Cambridge, Massachusetts 02139, USA \\ rcowan@mit.edu
}

Published 25 February 2015

\begin{abstract}
The clean environment of the $e^{+} e^{-}$collisions provided by the $B$-factory PEP-II and the high performances of the BABAR detector allow for precise measurements of the properties of inclusive hadron production. We present recent results obtained using the full data sample of nearly $500 \mathrm{fb}^{-1}$ on measurements of the inclusive light hadrons momentum spectra, and on anti-deuteron production from decays of bottomonium states, and from continuum $e^{+} e^{-}$annihilation. We also present a measurement of the azimuthal asymmetries induced by the Collins effect in inclusive production of pion pairs in $e^{+} e^{-} \rightarrow$ $\pi \pi X$ annihilation, where the two pions are produced in opposite hemispheres.
\end{abstract}

Keywords: inclusive hadron production; anti-deuteron; azimuthal asymmetries; Collins effect.

PACS numbers: 12.38.Qk, 13.25.Gv, 13.60.Rj, 13.66.Bc, 13.87.Fh, 13.88.+e, 14.65-q

\section{Introduction}

A fragmentation function ( $\mathrm{FF}$ ) describes a parton's hadronization process including non-perturbative effects. Studies using $e^{+} e^{-}$data provide the most direct access via $e^{+} e^{-} \rightarrow q \bar{q}$. The FF $D_{q}^{h}\left(z, Q^{2}\right)$ represents the probability that a parton $q$ fragments into a hadron $h$ with a fraction $z=2 E_{h} / \sqrt{s}$ of the original parton's momentum and relates the hadronic cross section to the partonic cross section:

$$
\sigma^{e^{+} e^{-} \rightarrow h X} \propto \sigma^{e^{+} e^{-} \rightarrow q \bar{q}} \times D_{q}^{h}\left(z, Q^{2}\right)
$$

Several phenomenological models have been developed that attempt to describe the hadronization process. Perturbative QCD (pQCD) corrections produce logarithmic scaling via the DGLAP evolution equations. Tests of scaling behavior require precise measurement of inclusive hadron production.

* Speaker on behalf of the BABAR Collaboration.

This is an Open Access article published by World Scientific Publishing Company. It is distributed under the terms of the Creative Commons Attribution 3.0 (CC-BY) License. Further distribution of this work is permitted, provided the original work is properly cited. 
FFs have been studied years, but most results are from higher energies (e.g., at LEP) than the $B$-factories and at lower $z$ values. There have been many attempts to extract FFs from $e^{+} e^{-}$data. ${ }^{1-5}$ Global analyses of $e^{+} e^{-}$, semi-inclusive deep inelastic scattering (SIDIS), and $p \bar{p}$ data have also been performed. ${ }^{6-8}$ More information is needed at lower energies and at higher $z$. The $B$-factory detectors are ideal for high-precision FF studies, providing high-resolution tracking, excellent particle identification, and uniform acceptance combined with large data sets of billions of events.

\section{Inclusive Hadron Production of $\pi^{ \pm}, K^{ \pm}, p / \bar{p}$}

This inclusive measurement uses $0.91 \mathrm{fb}^{-1}$ of $e^{+} e^{-} \rightarrow q \bar{q}(q=u, d, s, c)$ data taken at $\sqrt{s}=10.54 \mathrm{GeV}$, just below the $\Upsilon(4 S)$ resonance. An additional $3.61 \mathrm{fb}^{-1}$ on the $\Upsilon(4 S)$ are used for checks and calibrations. Results are systematics-dominated.

Event reconstruction starts with high-quality charged tracks obtained from multi-hadronic events originating at the primary interaction point (IP). Three or more charged tracks per event are required with a thrust axis well within the DIRC detector acceptance. Spectra are corrected for physics backgrounds of a few percent (especially $\tau^{+} \tau^{-}$), interaction in materials, efficiency and resolution effects.

Identification as pions, kaons or protons is performed using momentum and $d E / d x$ information from the drift chamber (DCH) and Cherenkov angle measurements from the DIRC detector. ${ }^{9}$ This provides good particle identification (PID) using the DCH from low to medium $p_{\text {lab }}$ (up to $0.5 \mathrm{GeV} / c$ for $\pi$ and $K, 0.8 \mathrm{GeV} / c$ for $K$ and $p / \bar{p}$ ), and using the DIRC up to $4 \mathrm{GeV} / c$ separation for $\pi$ and $K$ and $6.5 \mathrm{GeV} / c$ for $K$ and $p / \bar{p}$. PID performance is calibrated using control samples. High efficiency is obtained over much of the momentum range, with mis-identification probabilities on the order of a few percent.

Systematics are thoroughly studied along with many cross-checks. These include comparison to Monte Carlo (MC) simulation; angular dependencies; and charge dependencies. The largest effects arise from PID, backgrounds, and efficiencies.

We present results in terms of the scaled momentum $x_{p}=2 p_{\mathrm{cm}} / E_{\mathrm{cm}}$. in the momentum range $0.2 \mathrm{GeV} / c<5.27 \mathrm{GeV} / c$, and compare with three hadronization models using default parameters: UCLA, ${ }^{10}$ JETSET, ${ }^{11}$ and HERWIG. ${ }^{12,13}$ See Fig. 1 (left). We find overall qualitative agreement, but no one model describes the observed spectra in detail. Peak positions are consistent with the exception of HERWIG in the $K^{ \pm}$spectra.

We consider $\pi, K$, and $p$ results from BABAR, TASSO, and SLD, at $E_{\mathrm{cm}}$ values of $10.54 \mathrm{GeV}, 34 \mathrm{GeV}$, and $91.2 \mathrm{GeV}$, respectively. For pions, strong scaling violation is seen at high $x_{p}$ (running of $\alpha_{S}$ ) and at low $x_{p}$ (pion mass). For kaons, the differing composition of the three data samples modifies the expected scaling violation. The models predict about $10-15 \%$ more scaling violation than is observed. For protons, the scaling prediction for $10.54 \mathrm{GeV}$ is consistent with data for $x_{p}<0.07$, but exceeds it by as much as a factor of three at $x_{p} \approx 0.8$. See Fig. 1 (middle). 

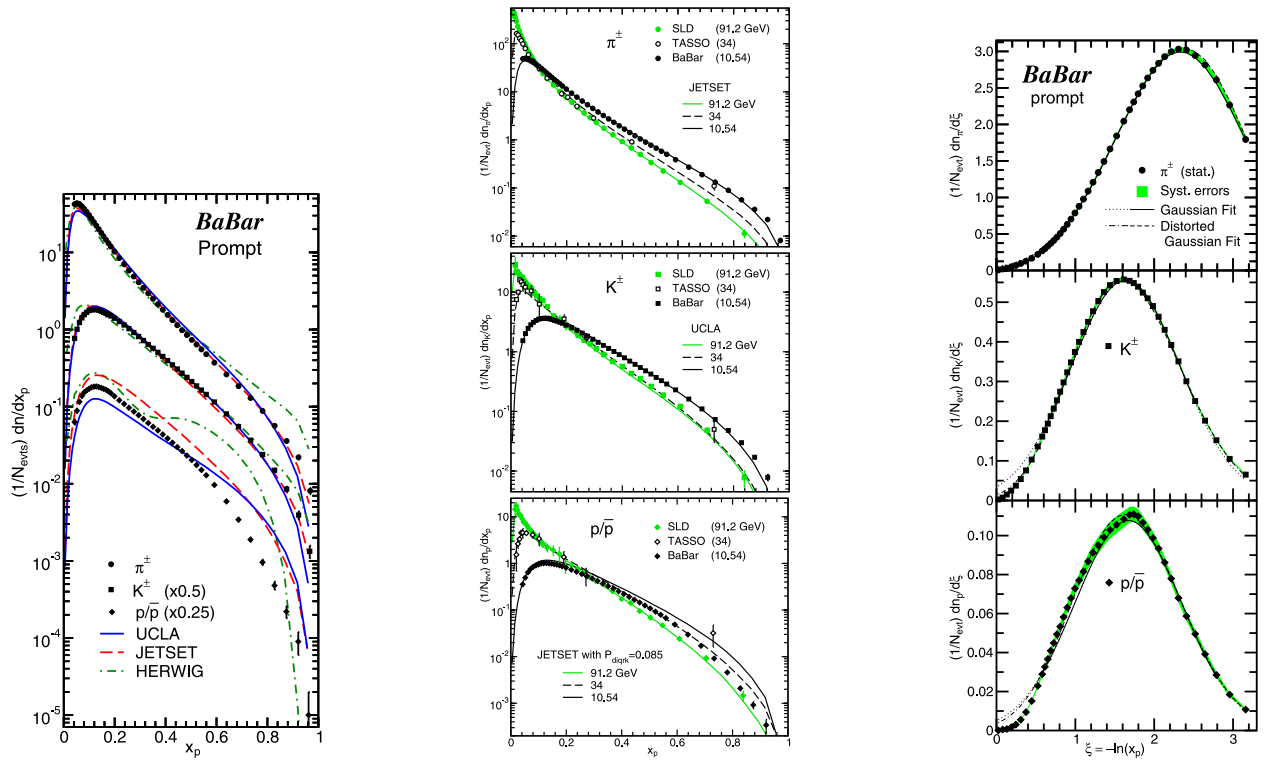

Fig. 1. Left: Cross sections of the prompt $\pi^{ \pm}$(top), $K^{ \pm}$(middle) and $p / \bar{p}$ (bottom) in $e^{+} e^{-} \rightarrow q \bar{q}$ events compared with the UCLA (solid line), JETSET (dashed) and HERWIG (dotted) hadronization models. Middle: Conventional $\pi^{ \pm}$(top), $K^{ \pm}$(middle) and $p / \bar{p}$ (bottom)cross sections measured at three different CM energies compared with the same models. Right: Differential cross sections in $\xi$ for prompt $\pi^{ \pm}$(top), $K^{ \pm}$(middle), and $p / \bar{p}$ (bottom). Error bars are statistical only; green bands represent the systematic uncertainties. Gaussian fits are shown (solid lines) and distorted Gaussian fits (dashed lines), with extensions outside their maximum range shown as dotted (Gaussian) and dash-dot (distorted Gaussian) lines.

Using the Modified Leading Log Approximation (MLLA) with Local PartonHadron Duality (LPHD), we expect that a Gaussian function should proved a good description of the inclusive cross sections when formulated in terms of the variable $\xi \equiv-\ln x_{p}$. In this situation, the peak position $\xi^{*}$ in each spectrum should decrease exponentially with increasing hadron mass at a given $E_{\mathrm{cm}}$, and should increase logarithmically with $E_{\mathrm{cm}}$ for a given hadron type. We fit the spectra in terms of $\xi$ with a distorted Gaussian function and find reasonable description of the data. We note that peak position $\xi_{\pi}^{*}$ is higher than $\xi_{K}^{*}$, in agreement with the predicted drop, but that $\xi_{p}^{*}$ is not lower than $\xi_{K}^{*}$. Similar behavior is observed at higher energies as well. See Fig. 1 (right).

\section{Collins Asymmetries Using Charged Pion Pairs}

Unpolarized FFs are now well known, using data from LEP, SLC, KEKB, and PEP-II. Collins ${ }^{14,15}$ proposed the use of single-particle distributions as a probe of a quark's transverse polarization. One looks for a spin asymmetry in the azimuthal distribution of the hadron about the jet axis. This can be described in terms of the Collins FF $H^{\perp}$. The FF $D_{q}^{h}\left(z, Q^{2}\right)$ can be expressed in terms of $H^{\perp}$, the decaying 

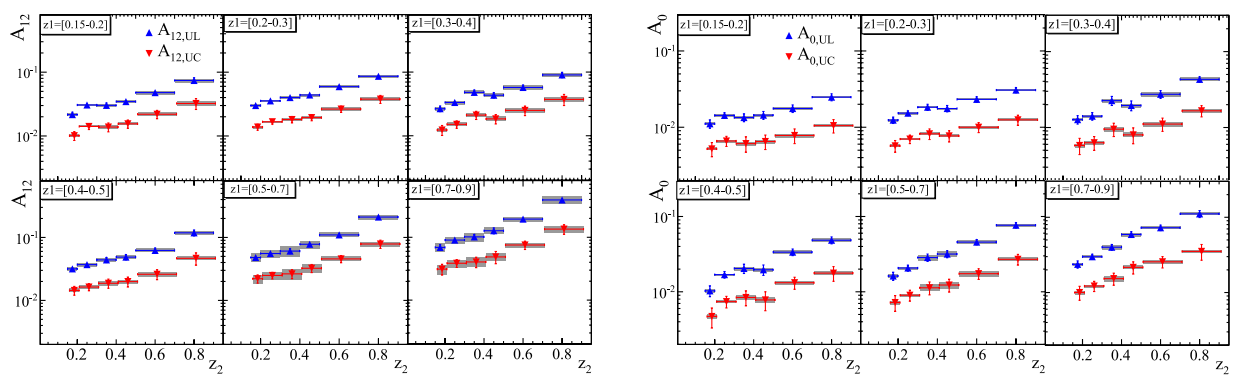

Fig. 2. Collins asymmetries for light quarks measured in bins of fractional energies $\left(z_{1}, z_{2}\right)$, in RF12 (upper) and RF0 (lower). Asymmetries for the UL (up triangles) and UC (down triangles) ratios are reported, with statistical error bars and systematic uncertainties represented by the bands around the points.
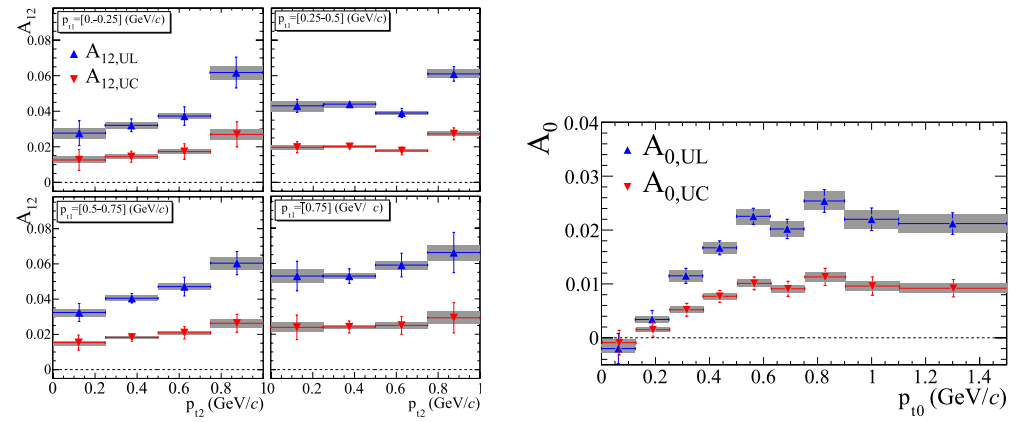

Fig. 3. Collins asymmetries for light quarks measured in $\left(p_{t 1}, p_{t 2}\right)$ bins in RF12 (left plots), and in nine bins of $p_{t 0}$ (right plot) in RF0. Asymmetries for the UL (up triangles) and UC (down triangles) ratios are reported, with statistical errors (bars) and systematic uncertainties (bands).

quark spin $\mathbf{S}_{q}$, and the hadron's transverse momentum $P_{h}^{\perp}$. This allows for an azimuthal asymmetry in $\phi$ of the hadrons around the quark momentum direction $\hat{\mathbf{k}}$, where $\phi$ is the angle between the plane perpendicular to $\mathbf{S}_{q}$ and that formed by $P_{h}^{\perp}$ and $\hat{\mathbf{k}}$. The amplitude of this asymmetry is the Collins effect.

We use two reference frames: the thrust frame (RF12) and the second-pion frame (RF0). In RF12, the event thrust axis is used to approximate the $q \bar{q}$ reference axis. In RF0, the direction of the second pion $\left(\pi_{2}\right)$ is used as the reference axis. The Collins asymmetry shows up in the azimuthal dependence of the final-state pions $\pi_{1}, \pi_{2}$, about the chosen axis. The rate depends on the angle $\phi_{\alpha}$, where $\phi_{\alpha}=\phi_{1}+\phi_{2}(\mathrm{RF} 12)$ or $\phi_{\alpha}=2 \phi_{0}$ (RF0). Divide $\phi_{\alpha}$ into bins and extract $R\left(\phi_{\alpha}\right)=N\left(\phi_{\alpha}\right) / N_{\text {average }}$. Events can be further classified into like-sign, unlike-sign, or charged pion pairs, giving access to FFs for favored and dis-favored decays.

Multi-hadronic events are selected by requiring $\geq 3$ high-quality charged tracks originating near the interaction point (IP). Events with energetic gluon emission, which disrupts the correlation between quark spins, are suppressed by requiring 

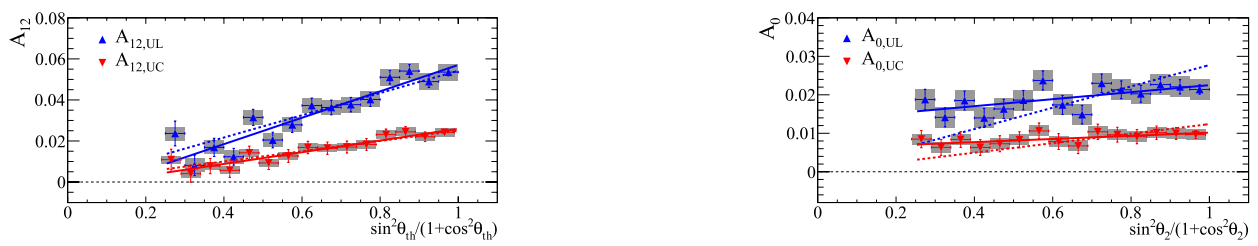

Fig. 4. Light quark asymmetry parameters calculated in RF12 (left) and in RF0 (right), as a function of $\sin ^{2} \theta /\left(1+\cos ^{2} \theta\right)$, where $\theta \equiv \theta_{t h}$ (left) and $\theta \equiv \theta_{2}$ (right). The up-triangles (blue online) refer to the UL asymmetry, while the down-triangles (red online) to the UC asymmetry. Systematic contributions are shown by gray boxes. The result of the linear fits are shown as solid lines of the corresponding colors. The dashed lines represent linear fits through the origin.
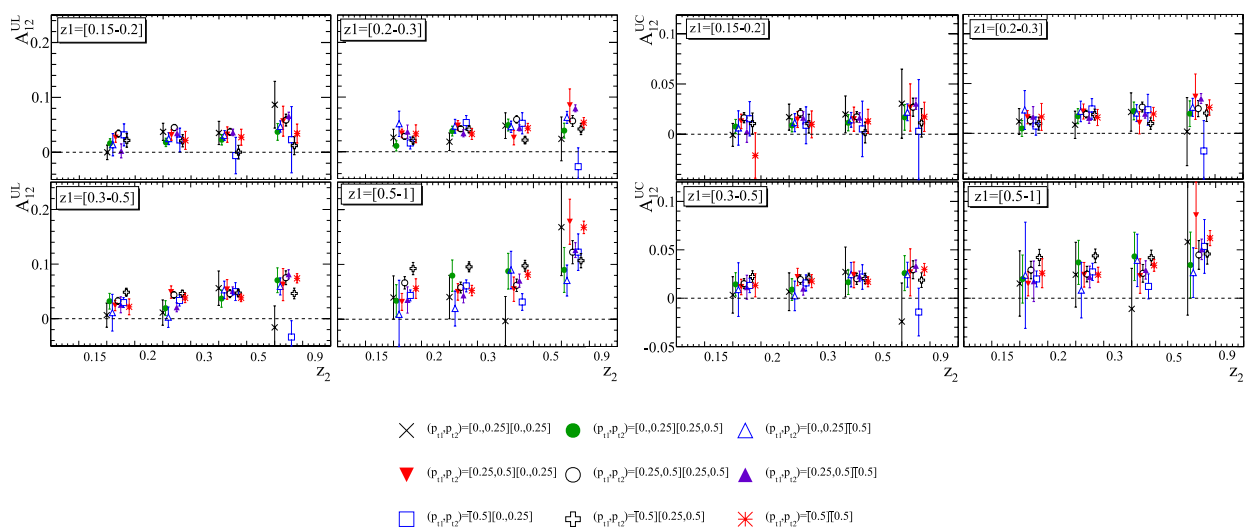

Fig. 5. Light quark asymmetry $A_{12}^{U L}$ (up) and $A_{12}^{U C}$ (down) calculated in the RF12 frame as a function of $\left(z_{1}, z_{2}, p_{t 1}, p_{t 2}\right)$. The plots show the $\left(z_{1}, z_{2}\right)$ dependence for each $\left(p_{t 1}, p_{t 2}\right)$ interval, identified by the different markers and colors as described in the legend.

the event thrust $T \geq 0.8$. Additional cuts and corrections using control samples suppress key backgrounds such as $e^{+} e^{-} \rightarrow \tau^{+} \tau^{-}, e^{+} e^{-} \rightarrow c \bar{c}, e^{+} e^{-} \rightarrow B \bar{B}, e^{+} e^{-} \rightarrow$ $\mu^{+} \mu^{-} \gamma$ and $e^{+} e^{-} \rightarrow e^{+} e^{-} \gamma$, resulting in a Collins analysis sample of $10^{8}$ pion pairs from $424 \mathrm{fb}^{-1}$ at the $\Upsilon(4 S)$ peak and $44 \mathrm{fb}^{-1}$ at $40 \mathrm{MeV}$ below. Detector acceptance effects create asymmetries in the cosine dependence of $\phi_{\alpha}$. These are largely eliminated by using ratios of like (UL), unlike (UU), and charged (UC) distributions: the double ratio method.

Asymmetry dilution effects due to use of the event thrust axis to approximate the $q \bar{q}$ axis, which has an average divergence of about $100 \mathrm{mrad}$, are accounted for by using Monte Carlo (MC) studies to determine the average dilution for each $z$, $p_{t}$, and $\theta_{\text {thrust }}$ bin which are then applied as corrections.

An approximation particularly useful for small values of the asymmetries is the double ratio $\frac{R_{\alpha}^{i}}{R_{\alpha}^{j}}=A_{\alpha}^{i j}+B_{\alpha}^{i j} \cdot \cos \left(\phi_{\alpha}\right)$. The Collins asymmetry is given by the value 
of $B_{\alpha}^{i j}$. The asymmetries depend only on the favored and disfavored FFs. Gluon radiation and acceptance are independent of pion pair charges.

The Collins asymmetry results for light quarks are presented using four different parameterizations. First, Fig. 2 shows results in terms of fractional energies $\left(z_{1}, z_{2}\right)$ for the RF12 and RF0 reference frames. Fig. 3 shows the asymmetries in terms of $\left(p_{t 1}, p_{t 2}\right)$ bins in RF12 and in bins of $p_{t 0}$ in RF0. This is the first measurement of Collins asymmetries as a function of transverse pion momenta. Results as a function of polar angle are given in Fig. 4. While the RF12 results have a linear fit intercept consistent with zero, as expected, the RF0 results do not. However, the RF0 frame is known to give less a less-accurate estimate of the $q \bar{q}$ direction. Finally, a fourdimensional presentation of the Collins asymmetries is shown in Fig. 5, in terms of $\left(z_{1}, z_{2}, p_{t 1}, p_{t 2}\right)$. This probes factorization of the Collins FFs.
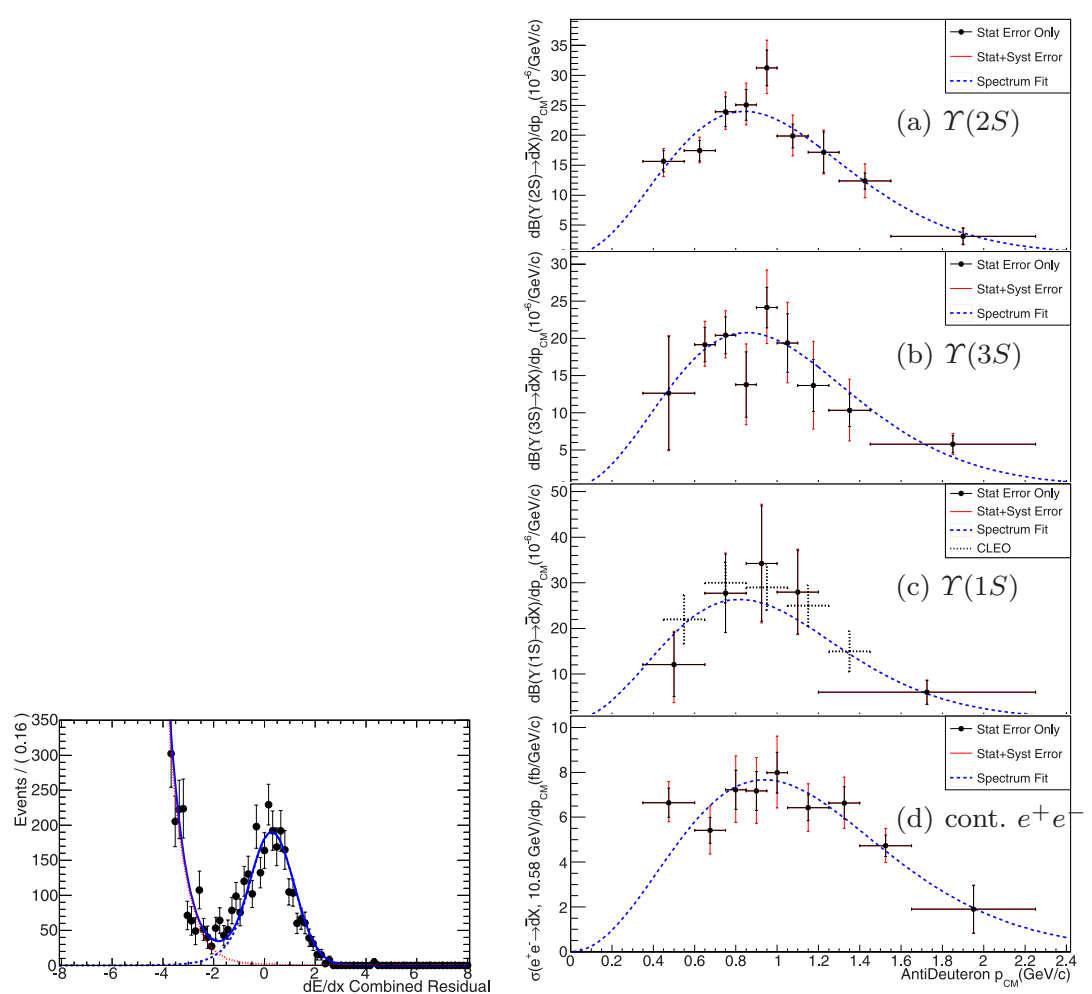

Fig. 6. Left: Normalized residuals of the combined $d E / d x$ for antideuteron candidates in the Onpeak $\Upsilon(2 S)$ data sample, with fit p.d.f.'s superimposed. The solid (blue) line is the total fit, the dashed (blue) line is the $\bar{d}$ signal peak, and the dotted (red) line is the background. Right: Measured antideuteron differential spectra in (a) $\Upsilon(2 S)$, (b) $\Upsilon(3 S)$, (c) $\Upsilon(1 S$ ) decays, and (d) $e^{+} e^{-} \rightarrow q \bar{q}$ at a $\mathrm{CM}$ energy of $\approx 10.58 \mathrm{GeV}$. Statistical uncertainties are shown as black error bars, with red error bars giving the quadratic sum of the statistical and systematic uncertainties. 
Table 1. Total rates of antideuteron production. The first uncertainties listed are statistical, the second systematic.

\begin{tabular}{lc}
\hline Process & Rate \\
\hline $\mathcal{B}(\Upsilon(3 S) \rightarrow \bar{d} X)$ & $\left(2.33 \pm 0.15_{-0.28}^{+0.31}\right) \times 10^{-5}$ \\
$\mathcal{B}(\Upsilon(2 S) \rightarrow \bar{d} X)$ & $\left(2.64 \pm 0.11_{-0.21}^{+0.26}\right) \times 10^{-5}$ \\
$\mathcal{B}(\Upsilon(1 S) \rightarrow \bar{d} X)$ & $\left(2.81 \pm 0.49_{-0.24}^{+0.20}\right) \times 10^{-5}$ \\
$\sigma\left(e^{+} e^{-} \rightarrow \bar{d} X\right)[\sqrt{s} \approx 10.58 \mathrm{GeV}]$ & $\left(9.63 \pm 0.41_{-1.01}^{+1.17}\right) f_{B}$ \\
\hline
\end{tabular}

\section{Anti-deuteron Production}

$e^{+} e^{-}$annihilations provide a clean probe of hadronization of quarks and gluons into anti-nuclei and are of interest in dark matter searches, providing a sensitive probe of dark matter annihilation. Anti-deuteron $\bar{d}$ production was observed in $\Upsilon(1 S)$ and $\Upsilon(2 S)$ decays by ARGUS ${ }^{16}$ and $\mathrm{CLEO}^{17}$ with a rate $\approx 3 \times 10^{-5}$. We update these measurements and also observe $\bar{d}$ production in $\Upsilon(3 S)$ decays and $e^{+} e^{-} \rightarrow q \bar{q}$ near $\sqrt{s}=10.6 \mathrm{GeV}$.

Event selection requires $\geq 3$ high-quality charged tracks in the DCH acceptance with $d E / d x$ values well away from those of other species. Additional cuts are also applied. Energy loss in the Silicon Vertex Tracker (SVT) is also used. Yields are extracted from combined DCH-plus-SVT dE/dx distributions. See Fig. 6 (left).

Systematics considered include the effects of fit biases, background model, reconstruction efficiency, kinematic acceptance, material interaction, fake anti-deuterons, selection cuts, and normalization. Results include correction of the number of $\bar{d}$ from efficiency effects. Total rates are computed from differential spectra. Differential spectra are shown in Fig. 6 (right). Rates are listed in Tab. 1

\section{Summary}

The BABAR Collaboration has measured inclusive production cross sections for $\pi^{ \pm}$, $K^{ \pm}$, and $p / \bar{p}$ spectra at center-of-mass energy $10.54 \mathrm{GeV}$ using a small, very highquality subsample of the full BABAR data set. ${ }^{18}$

Additionally, BABAR has measured Collins asymmetries in $e^{+} e^{-} \rightarrow q \bar{q}$ events using multi-hadron events with two pions in opposing jets; an analysis using kaons is underway. The asymmetries are extracted from ratios of normalized distributions of like-sign, unlike-sign, and charged pion pairs. Results are reported as a function of fractional energies, transverse pion momenta (first measurement), thrust axis polar angle, and the four-dimensional space $\left(z_{1}, z_{2}, p_{t 1}, p_{t 2}\right)$ (in RF12 reference frame only). Dependence of the asymmetries on $z_{i}$ and $p_{t}$ is in accord with expectations, but the polar angle dependence shows a difference from expectations. ${ }^{19}$

The anti-deuteron result is the most precise measurement in $\Upsilon(2 S)$ decays and is the first measurement in both $\Upsilon(3 S)$ decays and $e^{+} e^{-} \rightarrow q \bar{q}$ near $10.58 \mathrm{GeV} .^{20}$ 


\section{Acknowledgments}

We are grateful for the extraordinary contributions of our PEP-II colleagues in achieving the excellent luminosity and machine conditions that have made this work possible. The success of this project also relies on the expertise and dedication of the computing organizations that support BABAR. The collaborating institutions wish to thank SLAC for its support and the hospitality extended to them. This work is supported by the US Department of Energy and National Science Foundation, the Natural Sciences and Engineering Research Council (Canada), the Commissariat à l'Energie Atomique and Institut National de Physique Nucléaire et de Physique des Particules (France), the Bundesministerium für Bildung und Forschung and Deutsche Forschungsgemeinschaft (Germany), the Istituto Nazionale di Fisica Nucleare (Italy), the Foundation for Fundamental Research on Matter (The Netherlands), the Research Council of Norway, the Ministry of Education and Science of the Russian Federation, Ministerio de Economía y Competitividad (Spain), the Science and Technology Facilities Council (United Kingdom), and the Binational Science Foundation (U.S.-Israel). Individuals have received support from the Marie-Curie IEF program (European Union) and the A. P. Sloan Foundation (USA).

\section{References}

1. S. Albino, B. Kniehl and G. Kramer, Nucl.Phys. B725, 181 (2005).

2. S. Albino, B. Kniehl and G. Kramer, Nucl.Phys. B803, 42 (2008).

3. M. Hirai, S. Kumano, T.-H. Nagai and K. Sudoh, Phys.Rev. D75, p. 094009 (2007).

4. S. Kretzer, Phys.Rev. D62, p. 054001 (2000).

5. B. A. Kniehl, G. Kramer and B. Potter, Nucl.Phys. B582, 514 (2000).

6. D. de Florian, R. Sassot and M. Stratmann, Phys.Rev. D75, p. 114010 (2007).

7. D. de Florian, R. Sassot and M. Stratmann, Phys.Rev. D76, p. 074033 (2007).

8. M. Epele, R. Llubaroff, R. Sassot and M. Stratmann, Phys.Rev. D86, p. 074028 (2012).

9. B. Aubert et al., Nucl.Instrum.Meth. A729, 615 (2013).

10. S. Chun and C. Buchanan, Phys.Rept. 292, 239 (1998).

11. T. Sjostrand, Comput.Phys.Commun. 82, 74 (1994).

12. G. Corcella, I. Knowles, G. Marchesini, S. Moretti, K. Odagiri et al., JHEP 0101, p. 010 (2001).

13. G. Marchesini, B. Webber, G. Abbiendi, I. Knowles, M. Seymour et al., Comput.Phys.Commun. 67, 465 (1992).

14. J. C. Collins, Nucl.Phys. B396, 161 (1993).

15. J. C. Collins, Phys.Lett. B536, 43 (2002).

16. H. Albrecht et al., Phys.Lett. B236, p. 102 (1990).

17. D. Asner et al., Phys.Rev. D75, p. 012009 (2007).

18. J. Lees et al., Phys.Rev. D88, p. 032011 (2013).

19. J. Lees et al., Phys.Rev. D90, p. 052003 (2014).

20. J. Lees et al., Phys.Rev. D89, p. 111102 (2014). 